\title{
The Use of Psychiatric eConsults in Primary Care
}

\author{
Ezra Golberstein, Ph.D. ' ${ }^{7}$, Jennifer M. Joseph, Ph.D. ${ }^{7}$, Benjamin G. Druss, M.D., M.P.H. ${ }^{2}$,
} Hilary Carruthers, M.B.A., M.H.A. ${ }^{3}$, and Paul Goering, M.D.4

'Division of Health Policy and Management, University of Minnesota School of Public Health, Minneapolis, MN, USA; ${ }^{2}$ Department of Health Policy and Management, Rollins School of Public Health, Emory University, Atlanta, GA, USA; ${ }^{3}$, Children's Minnesota, Minneapolis, MN, USA; ${ }^{4}$ Mental

Health and Addiction Services, Allina Health, Minneapolis, MN, USA.

$\mathrm{J}$ Gen Intern Med 35(2):616-7

DOI: $10.1007 / \mathrm{s} 11606-019-05048-\mathrm{w}$

(c) Society of General Internal Medicine 2019

\section{INTRODUCTION}

Many mental health problems are identified, treated, and managed in primary care. But, primary care providers (PCPs) sometimes struggle to address patients' mental health conditions. Mental disorders are frequently undiagnosed, untreated, or inadequately managed in primary care, ${ }^{1}$ and PCPs report having poor access to mental health specialist consultation. ${ }^{2} \mathrm{~A}$ recent innovation, the electronic consultation (eConsult), may help alleviate these problems. eConsults are asynchronous consultations between providers, either over a shared electronic health record (EHR) system or via a web-based platform. An eConsult could support PCPs in delivering mental health care while avoiding specialty referrals, or could identify patients who acutely need a specialty referral. Psychiatrists may be well-situated to respond to eConsult requests because patient "no-shows" are relatively common. ${ }^{3}$

We add to the limited literature on eConsults for mental health care ${ }^{4-6}$ by assessing the adoption of psychiatric eConsults in a large integrated delivery system serving the Twin Cities region of Minnesota (Allina Health). Allina introduced psychiatric eConsults in 22 of its primary care clinics on August 1, 2015. A PCP would initiate an eConsult request through the EHR and eConsult requests were routed to Allina's psychiatrists in the corresponding service region. Psychiatrists would respond to eConsult requests in the order received. Patients had no fees for eConsults, and psychiatrists received 0.75 work relative value units for each eConsult

Prior Presentation This work has not been previously presented.

Received April 16, 2019

Accepted April 24, 2019

Published online May 14, 2019 completed. PCPs did not receive reimbursement for ordering eConsults.

\section{METHODS}

The data for these analyses were derived from Allina's EHR. We identified all primary care office visits for adults with an associated mental health-related diagnosis code, between August 1, 2015, and December 31, 2016. The sample included 95,105 encounters across 219 PCPs. We included variables for the type of PCP (MD, DO, NP, or $\mathrm{PA})$, whether the primary care clinic had co-located mental health services, encounter diagnoses and primary diagnosis flag, whether a psychiatric eConsult was ordered, and the date of the eConsult's response. Available patient characteristics were age, sex, race/ethnicity, and primary language. This study was approved by the IRBs of the University of Minnesota and Allina Health (which used Quorum IRB).

\section{RESULTS}

Only 256 of the primary care encounters $(0.27 \%)$ had a psychiatric eConsult order (Table 1). Among the 37,606 encounters with a primary mental health diagnosis, only $138(0.37 \%)$ had an eConsult order. There was no evidence of a time trend in eConsult use over the study period. Ninety-three percent of the eConsult requests had a response from the consulting psychiatrist that was sent within the EHR, and $86 \%$ of those responses occurred within 1 day. Anxiety disorders (66\%) and depressive disorders $(48 \%)$ were the most common diagnoses in primary care encounters with eConsult requests (Table 2). Anxiety disorders and depressive disorders were the most common diagnosis types for primary care encounters without an eConsult order, but the percentages were smaller than for encounters with an eConsult order. Schizophrenia and psychotic disorder diagnoses were relatively more common in encounters with an eConsult order, and bipolar disorder diagnoses were 
Table 1 Characteristics of the Sample

\begin{tabular}{lll}
\hline \hline & $\begin{array}{l}\text { Number } \\
\text { or mean }\end{array}$ & $\begin{array}{l}\text { Percent } \\
\text { or SD }\end{array}$ \\
\hline Female & 62,478 & $65.69 \%$ \\
Male & 32,627 & $34.31 \%$ \\
Age (mean) & 46.57 & 17.81 \\
Non-Hispanic White & 87,026 & $91.51 \%$ \\
Non-Hispanic Black & 2568 & $2.7 \%$ \\
Hispanic/Latino & 1615 & $1.7 \%$ \\
Asian/Pacific Islander & 1369 & $1.44 \%$ \\
American Indian/Alaskan Native & 483 & $0.51 \%$ \\
Other/not-specified & 2044 & $2.15 \%$ \\
Primary language is English & 94,234 & $99.08 \%$ \\
Primary language non-English or & 871 & $0.92 \%$ \\
not-specified & & \\
Primary visit diagnosis is MH & 37,574 & $39.51 \%$ \\
Multiple MH diagnoses in the visit & 20,801 & $21.87 \%$ \\
Total number diagnoses in the visit & 4.88 & 2.8 \\
(mean) & & \\
SUD diagnosis in the visit & 4603 & $4.84 \%$ \\
Non-physician PCP & 17,022 & $17.9 \%$ \\
Physician PCP & 78,083 & $82.1 \%$ \\
Co-located mental health & 74,656 & $78.5 \%$ \\
An eConsult was ordered & 256 & $0.27 \%$ \\
\hline
\end{tabular}

Source: Electronic health records from primary care office visits in 22 primary care clinics

relatively more common in encounters without an eConsult order. We also examined the reason for an eConsult request for 67 eConsult orders where that information was available to the researchers. Fifty-seven percent of those eConsults were for medication-related issues, 33\% were for a specific mental health diagnosis, and $15 \%$ were for psychiatry without specifying detail. The use of psychiatric eConsults was uneven across PCPs. Sixty-three percent of PCPs never ordered an eConsult, while the top ten users of eConsults accounted for $46 \%$ of the total eConsult orders.

Table 2 Percent of Primary Care Encounters with Specific Mental Health Diagnosis Categories for Primary Care Encounters with any Mental Health Diagnosis, With and Without an eConsult Request

\begin{tabular}{lll}
\hline \hline & $\begin{array}{l}\text { Primary care } \\
\text { encounters } \\
\text { with an } \\
\text { eConsult } \\
(\mathbf{N = 2 5 6 )}\end{array}$ & $\begin{array}{l}\text { Primary care } \\
\text { encounters } \\
\text { without an } \\
\text { eConsult } \\
\mathbf{( N = 9 4 , 9 4 9 )}\end{array}$ \\
\hline Mental illness category & & \\
Anxiety disorders & $66.4 \%$ & $58.1 \%$ \\
Depressive disorders & $48.0 \%$ & $38.3 \%$ \\
Adjustment disorders & $7.4 \%$ & $8.6 \%$ \\
Attention or conduct disorders & $7.0 \%$ & $7.5 \%$ \\
Childhood or adolescence disorders & $5.5 \%$ & $6.4 \%$ \\
Schizophrenia or psychotic & $5.1 \%$ & $1.2 \%$ \\
disorders & $2.3 \%$ & $0.4 \%$ \\
Personality disorders & $1.2 \%$ & $0.1 \%$ \\
Impulse control disorders & $0.4 \%$ & $3.8 \%$ \\
Bipolar disorders & $0.0 \%$ & $0.1 \%$ \\
Suicide or self-harm &
\end{tabular}

Source: Electronic health records from primary care office visits in 22 primary care clinics

\section{DISCUSSION}

As primary care practices and psychiatrists gain EHR capabilities, psychiatric eConsults can be a relatively low-cost and low-burden intervention to support and improve the delivery of mental health services in primary care. We found that the adoption of psychiatric eConsults by PCPs in a large integrated delivery system was relatively low, but that eConsults led to timely psychiatrist advice. It is unclear whether low eConsults use was due to unawareness of the tool, a perception that psychiatric eConsults were not useful, or that PCPs already feel overburdened and did not want to take additional time to engage with eConsults. Future research should assess the use and value of psychiatric eConsults in different clinical settings and aim to understand the barriers to using these services.

Corresponding Author: Ezra Golberstein, Ph.D.; Division of Health Policy and Management, University of Minnesota School of Public Health, Minneapolis, MN, USA (e-mail: egolber@umn.edu).

Funding This research was financially supported by research grants from the Agency for Healthcare Research and Quality (R03 HS25245O1) and Abbott Northwestern Hospital Fund (no. 15-8012). Dr. Golberstein was the PI on both grants.

\section{Compliance with Ethical Standards:}

This study was approved by the IRBs of the University of Minnesota and Allina Health (which used Quorum IRB).

Conflict of Interest: Ms. Carruthers and Dr. Goering were both employed by Allina Health when this research took place. The study authors affirm that they had access to all data from this study, both what is reported and unreported, and that had complete freedom in conducting and reporting the analysis without any influence from study sponsors. There was no editorial direction or censorship from study sponsors. Drs. Golberstein, Joseph, and Druss report no conflicts.

Prior Presentation: This work has not been previously presented.

\section{REFERENCES}

1. Kroenke K, Taylor-Vaisey A, Dietrich AJ, Oxman TE. Interventions to improve provider diagnosis and treatment of mental disorders in primary care. A critical review of the literature. Psychosomatics. 2000;41(1):39-52.

2. Cunningham PJ. Beyond parity: primary care physicians' perspectives on access to mental health care. Health Aff (Millwood). 2009;28(3):w490-501.

3. Dantas LF, Fleck JL, Cyrino Oliveira FL, Hamacher S. No-shows in appointment scheduling - a systematic literature review. Health Policy. 2018;122(4):412-421.

4. Golberstein E, Kolvenbach S, Carruthers H, Druss B, Goering P. Effects of electronic psychiatric consultations on primary care provider perceptions of mental health care: Survey results from a randomized evaluation. Healthc (Amst). 2018;6(1):17-22.

5. Lowenstein M, Bamgbose O, Gleason N, Feldman MD. Psychiatric Consultation at Your Fingertips: Descriptive Analysis of Electronic Consultation From Primary Care to Psychiatry. J Med Internet Res. 2017;19(8):e279.

6. Hensel JM, Yang R, Rai M, Taylor VH. Optimizing Electronic Consultation Between Primary Care Providers and Psychiatrists: Mixed-Methods Study. J Med Internet Res. 2018;20(4):e124.

Publisher's Note Springer Nature remains neutral with regard to jurisdictional claims in published maps and institutional affiliations. 